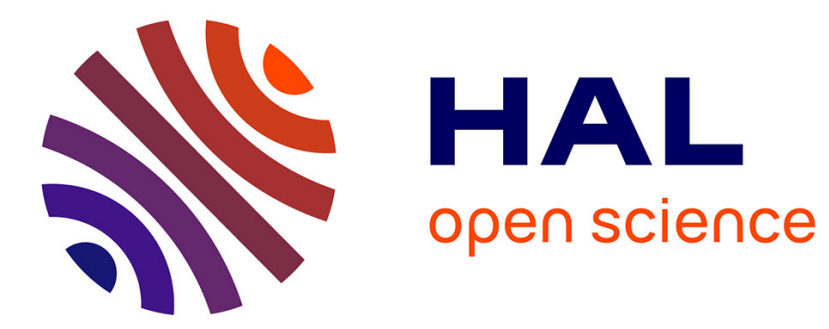

\title{
On the Semantics of Partially Ordered Bases
}

\author{
Claudette Cayrol, Didier Dubois, Fayçal Touazi
}

\section{To cite this version:}

Claudette Cayrol, Didier Dubois, Fayçal Touazi. On the Semantics of Partially Ordered Bases. 8th International Symposium on Foundations of Information and Knowledge Systems - FolKS 2014, Mar 2014, Bordeaux, France. pp. 136-153. hal-01147243

\section{HAL Id: hal-01147243 \\ https://hal.science/hal-01147243}

Submitted on 30 Apr 2015

HAL is a multi-disciplinary open access archive for the deposit and dissemination of scientific research documents, whether they are published or not. The documents may come from teaching and research institutions in France or abroad, or from public or private research centers.
L'archive ouverte pluridisciplinaire HAL, est destinée au dépôt et à la diffusion de documents scientifiques de niveau recherche, publiés ou non, émanant des établissements d'enseignement et de recherche français ou étrangers, des laboratoires publics ou privés. 


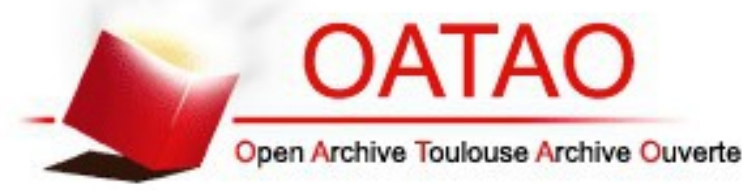

\section{Open Archive TOULOUSE Archive Ouverte (OATAO)}

OATAO is an open access repository that collects the work of Toulouse researchers and makes it freely available over the web where possible.

This is an author-deposited version published in : http://oatao.univ-toulouse.fr/ Eprints ID : 12863

To link to this article : DOI :10.1007/978-3-319-04939-7_6

URL : http://dx.doi.org/10.1007/978-3-319-04939-7 6

To cite this version : Cayrol, Claudette and Dubois, Didier and Touazi, Fayçal On the Semantics of Partially Ordered Bases. (2014) In: 8th International Symposium on Foundations of Information and Knowledge Systems - FolKS 2014, 3 March 2014 - 7 March 2014 (Bordeaux, France).

Any correspondance concerning this service should be sent to the repository administrator: staff-oatao@,listes-diff.inp-toulouse.fr 


\title{
On the Semantics of Partially Ordered Bases
}

\author{
Claudette Cayrol, Didier Dubois, and Fayçal Touazi \\ IRIT, University of Toulouse, France \\ \{ccayrol, dubois, faycal.touazi\}eirit.fr
}

\begin{abstract}
This paper presents first results toward the extension of possibilistic logic when the total order on formulas is replaced by a partial preorder. Few works have dealt with this matter in the past but they include some by Halpern, and Benferhat et al. Here we focus on semantic aspects, namely the construction of a partial order on interpretations from a partial order on formulas and conversely. It requires the capability of inducing a partial order on subsets of a set from a partial order on its elements. The difficult point lies in the fact that equivalent definitions in the totally ordered case are no longer equivalent in the partially ordered one. We give arguments for selecting one approach extending comparative possibility and its preadditive refinement, pursuing some previous works by Halpern. It comes close to non-monotonic inference relations in the style of Kraus Lehmann and Magidor. We define an intuitively appealing notion of closure of a partially ordered belief base from a semantic standpoint, and show its limitations in terms of expressiveness, due to the fact that a partial ordering on subsets of a set cannot be expressed by means of a single partial order on the sets of elements. We also discuss several existing languages and syntactic inference techniques devised for reasoning from partially ordered belief bases in the light of this difficulty. The long term purpose is to find a proof method adapted to partially ordered formulas, liable of capturing a suitable notion of semantic closure.
\end{abstract}

\section{Introduction}

The basic concept of ordered knowledge base expressing the relative strength of formulas has been studied for more than twenty years in Artificial intelligence. To our knowledge this concept goes back to Rescher's work on plausible reasoning [1]. The idea of reasoning from formulas of various strengths is even older, since it goes back to antiquity with the texts of Theophrastus, a disciple of Aristotle, who claimed that the validity of a chain of reasoning is the validity of its weakest link. Possibilistic logic [2] is a typical example of logic exploiting a totally ordered base and implementing the weakest link principle. It is an extension of propositional logic, sound and complete with respect to a semantics in terms of possibility theory, where a set of models is replaced by a possibility distribution on the interpretations (which are then more or less plausible). It enables problems of inconsistency management [3], of revision [4] and of information fusion [5] to be handled in a natural way.

This simple approach has limitations in expressive power. We may go beyond it in several respects: 
- extending the syntax to give a meaning to negations and disjunctions of weighted formulas, thus joining the syntactic framework of modal logic [6];

- improving the treatment of the degrees attached to the formulas via a refinement of the induced possibility distribution, possibly by means of a partial order [3];

- making the approach more qualitative, by replacing the weights of certainty by the elements of a lattice, or by a partial order over a finite set of formulas [7].

This paper paves the way to the systematic study of the last two points, based on scattered existing works. Possibilistic logic exploits the equivalence between the deductive closure of a set of weighted formulas, and a possibility distribution on the interpretations. In case of logical inconsistency, it reasons with the formulas whose certainty level exceeds the global inconsistency degree, leaving out some highly uncertain formulas not concerned by inconsistency (this is called "the drowning effect"). Our idea is to preserve this kind of relation between semantics and syntax in the setting of weaker algebraic frameworks (with partial preorders), while proposing concepts of partially ordered closure accordingly. The following questions seem natural:

- Is it possible to represent a partially ordered set of formulas by a partially ordered set of models?

- Is it possible to represent a partially ordered set of models by a partially ordered set of formulas?

- Is it possible to define inference rules that account for such a semantics?

To address these questions, we first review how to go from a partial ordering on a set to a partial ordering on its subsets. This point, already reviewed by Halpern [8], is tricky because equivalent definitions in the case of a total order are no longer so in the partially ordered setting. Properties of partial orders among sets induced by partial order on elements are studied in detail. Then these results are applied to the definition of semantic inference from partially ordered knowledge bases. This definition poses the problem of representing the semantics of a partially ordered base in terms of a single partial order of its interpretations. We show that in general the partial order on formulas cannot be recovered from the partial order on interpretations it induces, contrary to the totally ordered case. Finally we briefly review existing proposals of syntactic inference that may be used to reason from partially ordered formulas, in the light of this limitation.

\section{Comparing sets of totally ordered elements}

Let $(S, \geq)$ be a totally ordered set and let $A$ and $B$ be subsets of $S$. To extend $\geq$ to $2^{S}$, a natural idea is to compare $A$ with $B$ by means of logical quantifiers. So, four kinds of relations can be defined:

\section{Definition 1}

- Unsafe dominance: $A \succeq_{u} B$ iff $\exists a \in A, b \in B, a \geq b$

- Optimistic dominance: $A \succeq_{o} B$ iff $\forall b \in B, \exists a \in A, a \geq b$

- Pessimistic dominance: $A \succeq_{p} B$ iff $\forall a \in A, \exists b \in B, a \geq b$

- Safe dominance: $A \succeq_{s} B$ iff $\forall a \in A, \forall b \in B, a \geq b$ 
Strict counterparts of these definitions, namely $\succ_{u}, \succ_{o}, \succ_{p}, \succ_{s}$ can be similarly defined, replacing $(S, \geq)$ by its strict part $(S,>)$

Note that relations $\succ_{x}$ are not the strict parts of $\succeq_{x}$. The four kinds of weak relations in this definition can be rewritten by comparing their maximal (resp. minimal) elements. Denoting by $\max (A)$ (resp. $\min (A))$ any maximal (resp. minimal) element in $A$ :

- Unsafe dominance: $A \succeq_{u} B$ iff $\max (A) \geq \min (B)$

- Optimistic dominance: $A \succeq_{o} B$ iff $\max (A) \geq \max (B)$

- Pessimistic dominance: $A \succeq_{p} B$ iff $\min (A) \geq \min (B)$

- Safe dominance: $A \succeq_{s} B$ iff $\min (A) \geq \max (B)$

Note that $A \succeq_{u} B$ iff $\neg\left(B \succ_{s} A\right)$. The strict safe dominance $\succ_{s}$ is a strict partial order that can compare disjoint sets only; and $\succeq_{s}$ is not even reflexive. The unsafe dominance is not transitive even if reflexive. The optimistic and pessimistic comparisons are total orders dual to each other in the following sense: $A \succeq_{o} B$ iff $B \succeq_{p}^{\prime} A$ where $\geq^{\prime}$ denotes the inverse of $\geq$ on $S$, defined by $a \geq^{\prime} b$ iff $b \geq a$. It is interesting to highlight the point that the latter comparisons can be defined equivalently as:

- Optimistic dominance: $A \succeq_{o} B$ iff $\exists a \in A, \forall b \in B, a \geq b$;

- Pessimistic dominance: $A \succeq_{p} B$ iff $\exists b \in B, \forall a \in A, a \geq b$

These notions can be applied to the representation of uncertainty. Let $S$ denote a set of states. Assume $\pi$ a possibility distribution on $S$ such that $\pi(s)$ is the plausibility degree that $s$ is the real world. Let $\Pi$ the associated possibility measure defined by $\Pi(A)=\max _{s \in A} \pi(s)$ and $N$ the dual necessity measure defined by $N(A)=$ $\min _{s \notin A} 1-\pi(s)=1-\Pi(\bar{A})[2]$. We have:

- $\Pi(A) \geq \Pi(B)$ iff $\max (A) \geq \max (B)$ (this is $\left.A \succeq_{o} B\right)$

- $N(A) \geq N(B)$ iff $\max (\bar{B}) \geq \max (\bar{A})$ (this is $\left.\bar{B} \succeq_{0} \bar{A}\right)$

So the optimistic comparison between $A$ and $B$ is a comparative possibility measure in the sense of Lewis [9] (see also [10]), and the optimistic comparison between $\bar{A}$ and $\bar{B}$, which expresses relative certainty, is related to epistemic entrenchment in revision theory [11]. In the uncertainty framework, safe dominance is never used as it is not representable by a monotonically increasing set function. On the other hand, the pessimistic ordering is monotonically decreasing with inclusion. In the following, we thus concentrate on the optimistic comparison $\succeq_{0}$.

The above definitions also apply to the representation of preferences. Then stating $A \succeq B$ accounts for an agent declaring that the truth of the proposition whose models form the set $A$ is preferred to the truth of the proposition whose models form the set $B$. Interpreting such a statement requires the knowledge of the attitude of the agent, which leads to choosing between the four orderings considered above. The safe dominance is natural in this setting as a very conservative risk-free understanding of $A \succeq B$, akin to interval orderings [12]. Pessimistic and optimistic dominance are milder views, and both make sense, as explored by Benferhat et al. [13], Kaci and van den Torre [14, 15], contrary to the case of representing the plausibility and certainty of formulas. However, preference modelling is not in the scope of this paper. 


\section{Properties of relative likelihood relations comparing subsets}

Let $\unrhd$ be a reflexive relation that compares subsets $A$ and $B$ of $S$, and $\triangleright$ its strict part. We enumerate different properties that may be satisfied by these relations in the scope of modeling relative uncertainty.

1. Compatibility with set-theoretic operations (inclusion, intersection, union)

- Compatibility with Inclusion (CI) If $B \subseteq A$ then $A \unrhd B$

- Orderliness (O) If $A \triangleright B, A \subseteq A^{\prime}$, and $B^{\prime} \subseteq B$, then $A^{\prime} \triangleright B^{\prime}$

- Stability for Union (SU) If $A \unrhd B$ then $A \cup C \unrhd B \cup C$

- Preadditivity (P) If $A \cap(B \cup \bar{C})=\varnothing$ then $(B \unrhd C$ iff $A \cup B \unrhd A \cup C$ )

- Self-duality (D) $A \unrhd B$ iff $\bar{B} \unrhd \bar{A}$

Note that $(\mathrm{CI})$ is never satisfied by a non-reflexive relation while $(\mathrm{O})$ makes sense for a reflexive relation too. (SU) does not make sense for an asymmetric relation (take $C=S$ ). Preadditivity and self-duality, like (O) make sense for both $\unrhd$ and its strict part. All these properties have been studied in the case of total orders: (CI) and $(\mathrm{O})$ are expected when $A \triangleright B$ expresses a greater confidence in $A$ than in $B$; (SU) characterizes possibility relations $\succeq_{o}$ (Lewis[9], Dubois[10]). Preadditivity and self-duality hold for probability measures [16], but also for the relation $A \triangleright B$ iff $A \backslash B \succ_{o} B \backslash A$ [17].

2. Properties reflecting a qualitative point of view

- Qualitativeness (Q) If $A \cup B \triangleright C$ and $A \cup C \triangleright B$, then $A \triangleright B \cup C$

- Negligibility (N) If $A \triangleright B$ and $A \triangleright C$, then $A \triangleright B \cup C$

(Q) is satisfied by strict parts of possibility relations $\left(\succ_{o}\right.$, but not $\left.\succeq_{o}\right)$ and is found in non-monotonic logic. Negligibility also works for $\succeq_{o}$, it says that one cannot compensate for the low plausibility of a set by adding elements of low plausibility.

3. Properties concerning the deductive closure of partially ordered bases (see Friedman and Halpern[18], Dubois and Prade [19] and Halpern [8]):

- Conditional Closure by Implication (CCI) If $A \subseteq B$ and $A \cap C \triangleright \bar{A} \cap C$ then $B \cap C \triangleright \bar{B} \cap C$

- Conditional Closure by Conjunction (CCC) If $C \cap A \triangleright C \cap \bar{A}$ and $C \cap B \triangleright$ $C \cap \bar{B}$ then $C \cap(A \cap B) \triangleright C \cap \overline{A \cap B}$

- Left Disjunction (OR) If $A \cap C \triangleright A \cap \bar{C}$ and $B \cap C \triangleright B \cap \bar{C}$ then $(A \cup B) \cap$ $C \triangleright(A \cup B) \cap \bar{C}$

- Cut (CUT) If $A \cap B \triangleright A \cap \bar{B}$ and $A \cap B \cap C \triangleright A \cap B \cap \bar{C}$ then $A \cap C \triangleright A \cap \bar{C}$

- Cautious Monotony (CM): If $A \cap B \triangleright A \cap \bar{B}$ and $A \cap C \triangleright A \cap \bar{C}$ then $A \cap B \cap C \triangleright A \cap B \cap \bar{C}$

These properties are rather intuitive when the relation $A \triangleright \bar{A}$ is interpreted as " $A$ is an accepted belief", and $A \cap C \triangleright \bar{A} \cap C$ as " $A$ is an accepted belief in the context $C$ " $[20,18,21]$. They hold in the total order setting for the optimistic relation $\succ_{o}$, but they are not interesting to consider for reflexive relations (e.g. $\succeq_{o}$ ).

Proposition 1 It is easy to see that, for any relation $\gg$ :

1. (O) implies $C C I$.

2. If the relation $\gg$ is qualitative $(Q)$ and orderly $(O)$, then it satisfies Negligibility $(N)$ and $(C C C)$, and the converse of (SU): If $A \cup C \gg B \cup C$ then $A \gg B$. 


\section{Proof:}

1. Suppose $A \subseteq B$ and $A \cap C \gg \bar{A} \cap C$. We have $A \cap C \subseteq B \cap C$ and $\bar{B} \cap C \subseteq \bar{A} \cap C$. Hence from (O), $B \cap C \gg \bar{B} \cap C$.

2. Assume that $\gg$ satisfies $(\mathrm{Q})$ and $(\mathrm{O})$.

Suppose $A \gg B$ and $A \gg C$. Then, by (O), $A \cup B \gg C$ and $A \cup C \gg B$. Hence from $(\mathrm{Q}), A \gg B \cup C$. So $\gg$ satisfies $(\mathrm{N})$.

Suppose $C \cap A \gg C \cap \bar{A}$ and $C \cap B \gg C \cap \bar{B}$. Let $A^{\prime}=A \cap B \cap C, B^{\prime}=A \cap C \cap \bar{B}$, $C^{\prime}=\bar{A} \cap B \cap C$ and $D^{\prime}=C \cap \bar{B} \cap \bar{A}$. So, we have $A^{\prime} \cup B^{\prime} \gg C^{\prime} \cup D^{\prime}$ and $A^{\prime} \cup C^{\prime} \gg B^{\prime} \cup D^{\prime}$. Hence from $(\mathrm{O}), A^{\prime} \cup B^{\prime} \cup D^{\prime} \gg C^{\prime}$, then from (Q) $A^{\prime} \gg B^{\prime} \cup C^{\prime} \cup D^{\prime}$. So, $A \cap B \cap C \unrhd C \cap(\bar{A} \cup \bar{B})=C \cap \overline{A \cap B}$. So $\gg$ satisfies (CCC).

Suppose $A \cup C \gg B \cup C$. Then by (O), $A \cup(C \cup B) \gg C$. Hence from (Q), $A \gg B \cup C$, then (O) again: $A \gg B$. So $\gg$ satisfies the converse of (SU).

\section{Comparing sets of partially ordered elements}

In this section, we start from a partially ordered set $(S, \geq)$ and we consider the construction of a relation $\unrhd$

induced by $\geq$ for comparing subsets of $S$. In the scope of representing comparative belief and plausibility, the last section has shown that we can restrict to the optimistic comparison of sets. In the following, we focus on the generalization of optimistic dominance to the case of partially ordered sets. It has been noticed (see section 2) that there are two possible definitions of the

optimistic dominance, that are equivalent in the total order setting. However they are no longer so in the partial order setting, as first noticed by Halpern [8]. As a consequence, in order to define a semantics for partially ordered logical bases, we have to study these different relations and to choose an appropriate one according to the properties they satisfy.

As usual, given a reflexive and transitive relation on $S$, denoted by $\geq, s^{\prime}>s$ is an abbreviation for " $\left(s^{\prime} \geq s\right)$ and not $\left(s \geq s^{\prime}\right)$ ". The relation $>$ is the strict partial order determined by $\geq$. It is an irreflexive and transitive relation on $S . s^{\prime} \sim s$ is an abbreviation for " $\left(s^{\prime} \geq s\right)$ and $\left(s \geq s^{\prime}\right)$ ". The relation $\sim$ is the equivalence relation determined by $\geq . s^{\prime} \not \approx$ is an abbreviation for "(neither $\left(s^{\prime} \geq s\right)$ nor $\left(s \geq s^{\prime}\right)$ ". It is the incomparability relation determined by $\geq$. If this relation is empty, the relation $\geq$ is a total preorder. On the contrary, given a transitive and asymetric relation $>$, the relation $s^{\prime} \not \approx s$ if and only if neither $s^{\prime}>s$ nor $s>s^{\prime}$ is its associated incomparability relation (while $s^{\prime} \sim s$ reduces to the equality relation).

Let $(S, \geq)$ a partially ordered set, and $X \subseteq S . s \in X$ is maximal for $\geq$ in $X$ if and only if we do not have $s^{\prime}>s$ for any $s^{\prime} \in X . M(X, \geq)(M(X)$ for short) denotes the set of the maximal elements in $X$ according to $\geq$.

The optimistic comparison between $A$ and $B$ is based on the comparison between $M(A)$ and $M(B)$. In the total order case, it can be defined in two ways, which are no longer equivalent in the partial case. We call them weak optimistic dominance and strong optimistic dominance in the following. 


\subsection{Weak optimistic dominance}

Here again, various definitions can be proposed according to whether one starts from a strict order or not on $S$.

\section{Definition 2 (Weak optimistic dominance)}

1. Weak optimistic strict dominance:

$A \succ_{\text {wos }} B$ iff $A \neq \varnothing$ and $\forall b \in B, \exists a \in A, a>b$.

2. Weak optimistic loose dominance: $A \succeq_{\text {wol }} B$ iff $\forall b \in B, \exists a \in A, a \geq b$.

3. Strict order determined by $\succeq_{\text {wol }}: A \succ_{\text {wol }} B$ iff $A \succeq_{\text {wol }} B$ and $\neg\left(B \succeq_{\text {wol }} A\right)$. In other words, $A \succ_{\text {wol }} B$ iff $\forall b \in B, \exists a \in A, a \geq b$ and $\exists a^{\prime} \in A, \forall b \in$ $B$, either $a^{\prime}>b$ or $a^{\prime} \not \varpi b$.

These relations are respectively denoted by $\succ^{s}, \succeq^{s}$ and $\succ^{\prime}$ by Halpern [8]. The relation $\succ_{\text {wos }}$ is a strict partial order (asymetric and transitive) on $2^{S}$. We have always $A \succ_{\text {wos }} \varnothing$, except if $A$ is empty. The relation $\succeq_{\text {wol }}$ is reflexive and transitive and such that $A \succeq_{\text {wol }} \varnothing$, but not $\varnothing \succeq_{\text {wol }} B$ except if $B$ is empty. Finally, if $A \succ_{\text {wos }} B$ then $A \succ_{\text {wol }} B$. The converse is generally false except if $\geq$ is a complete order.

The following proposition shows that the weak optimistic dominance is appropriate for representing relative plausibility.

Proposition 2 The weak optimistic strict dominance $\succ_{\text {wos }}$ is a strict partial order which satisfies Qualitativeness $(Q)$, Orderliness $(O)$, Left Disjunction (OR), (CUT) and $(C M)$.

The weak optimistic loose dominance $\succeq_{\text {wol }}$ satisfies Compatibility with inclusion (CI), Orderliness $(O)$, Negligibility $(N)$, Stability for union $(S U)$.

The relation $\succ_{\text {wol }}$ satisfies Orderliness $(O)$ and Conditional Closure by Implication $(C C I)$.

Corollary 1 The weak optimistic strict dominance $\succ_{\text {wos }}$ satisfies the converse of (SU), Negligibility (N), Conditional Closure by Implication (CCI) and Conditional Closure by Conjunction (CCC).

Note that, as shown by Halpern [8], the relation $\succeq_{\text {wol }}$ is generally not qualitative and the relation $\succ_{w o l}$ does not satisfy the property of Negligibility. Moreover, the relation $\succ_{\text {wol }}$ neither satisfies the property OR nor the property CUT as shown below:

Example 1 Let $S=\{a, b, c, d, e, f, g, h\}$ be a partially ordered set with $f \sim h, e \sim$ $g, f>a, e>b, a>c$ and $b>d$.

OR : Let $A=\{a, c, e, g\}, B=\{b, d, f, h\}$ and $C=\{a, b, e, f\}$ be three subsets of $S$. We have $A \cap C \succ_{\text {wol }} A \cap \bar{C}$ and $B \cap C \succ_{\text {wol }} B \cap \bar{C}$ but not $(A \cup B) \cap C \succ_{\text {wol }}$ $(A \cup B) \cap \bar{C}$.

CUT : Let $A=\{a, b, c, d, e, f, g, h\}, B=\{a, b, c, e, g, h\}$ and $C=\{a, b, d, g, h\}$ be three subsets of $S$.

We have $A \cap B \succ_{\text {wol }} A \cap \bar{B}$ and $A \cap B \cap C \succ_{\text {wol }} A \cap B \cap \bar{C}$ but not $A \cap C \succ_{\text {wol }} A \cap \bar{C}$.

It is clear that as a result, the relation $\succ_{\text {wos }}$ is the richest one to represent relative plausibility. But note that it has no non trivial associated equivalence relation (but for $A \sim_{\text {wos }} B$ if and only if $A=B$ ). 


\subsection{Strong optimistic dominance}

The alternative approach, not considered by [8], consists in assuming, if $A \triangleright B$, that one element in $A$ dominates all elements in $B$. As before, various definitions can be proposed according to whether one uses a strict order or not on $S$.

\section{Definition 3 (Strong optimistic dominance)}

1. Strong optimistic strict dominance: $A \succ_{\text {Sos }} B$ iff $\exists a \in A, \forall b \in B, a>b$

2. Strong optimistic loose dominance: $A \succeq_{\text {Sol }} B$ iff $\exists a \in A, \forall b \in B, a \geq b$

3. Strict order determined by $\succeq_{\text {Sol }}: A \succ_{\text {Sol }} B$ iff $A \succeq_{\text {Sol }} B$ and $\neg\left(B \succeq_{\text {Sol }} A\right)$. In other words, $A \succ_{\text {Sol }} B$ iff $\exists a \in A, \forall b \in B, a \geq b$ and $\forall b \in B, \exists a \in$ $A$, either $a>b$ or $a \not z b$.

Note that with the above definitions, if $A \neq \varnothing, A \succ_{\text {Sos }} \varnothing$ and never $\varnothing \succ_{\text {Sos }} B$. The relation $\succ_{\text {Sos }}$ is a strict partial order on $2^{S}$. Finally, if $A \succ_{\text {Sos }} B$ then $A \succ_{\text {Sol }} B$. Obviously, the strong relations are stronger than the weak relations, namely: If $A \succ$ Sos $B$ then $A \succ_{\text {wos }} B$ and if $A \succeq_{S o l} B$ then $A \succeq_{w o l} B$. The converse is true only if $\geq$ is a complete order on $S$. So, we also have: If $A \succ_{\text {Sos }} B$ then $A \succ_{\text {wol }} B$. However there is no entailment between the relations $\succ_{\text {Sol }}$ and $\succ_{\text {wol }}$ as shown by the following counterexamples:

Example 2 Let $S=\left\{a_{1}, a_{2}, b_{1}, b_{2}, b_{3}\right\}$ with $a_{1} \sim b_{1}>b_{3}$ and $a_{2}>b_{2}$. Then $\left\{a_{1}, a_{2}\right\} \succ_{\text {wol }}\left\{b_{1}, b_{2}, b_{3}\right\}$, but it is false that $\left\{a_{1}, a_{2}\right\} \succ_{\text {Sol }}\left\{b_{1}, b_{2}, b_{3}\right\}$.

Example 3 Let $S=\left\{a_{1}, a_{2}, b_{1}, b_{2}, b_{3}\right\}$ with $a_{1} \sim b_{1}>b_{3}$, and $a_{1}>b_{2}>a_{2}$. Then $\left\{a_{1}, a_{2}\right\} \succ_{\text {Sol }}\left\{b_{1}, b_{2}, b_{3}\right\}$, but it is false that $\left\{a_{1}, a_{2}\right\} \succ_{\text {wol }}\left\{b_{1}, b_{2}, b_{3}\right\}$.

As indicated by Benferhat, Lagrue, Papini [22], the relation $\succ_{\text {Sos }}$ contains many incomparabilities, and $\succeq_{S o l}$ does not satisfy Compatibility with Inclusion. Indeed, if $A \subseteq B$, it is not obvious that there exists $b \in B$ such that $b \geq a, \forall a \in A$. In fact, $\succeq_{\text {Sol }}$ is thus not even reflexive, even if it is transitive. Finally, $A \succ_{\text {Sos }} B$ implies $A \succ_{\text {Sol }} B$. The converse is not true except when $\geq$ is a complete order on $S$. As for properties:

Proposition 3 The strong optimistic strict dominance $\succ_{\text {Sos }}$ is a strict order satisfying Orderliness $(O)$ and Cautious Monotony $(C M)$

However it fails to satisfy Negligibility, Qualitativeness, CUT and Left Disjunction (OR), as shown by the following examples.

Example 4 Let $S=\left\{a_{1}, a_{2}, b, c\right\}$ with $a_{1}>b$ and $a_{2}>c$, and the subsets $A=$ $\left\{a_{1}, a_{2}\right\}, B=\{b\}$ and $C=\{c\}$. We have $A \succ_{\text {Sos }} B, A \succ_{\text {Sos }} C$ but we don't have $A \succ_{\text {Sos }}(B \cup C)$. So $(N)$ is not satisfied.

Due to Proposition 1, the relation $\succ_{\text {Sos }}$ fails to satisfy Qualitativeness as well.

Example 5 Let $S=\{a, b, c, d\}$ with $a>b$ and $c>d$, and the subsets $A=\{a, b, c, d\}, B=$ $\{a, c, d\}$ and $C=\{a, c\}$. We have $A \cap B \succ_{\text {Sos }} A \cap \bar{B}, A \cap B \cap C \succ_{\text {Sos }} A \cap B \cap \bar{C}$ but we don't have $A \cap C \succ_{\text {Sos }} A \cap \bar{C}$. So $(C U T)$ is not satisfied. 
Lastly, suppose $\exists a \in A \cap C, \forall x \in A \cap \bar{C} a>x$, and $\exists b \in B \cap C, \forall x \in B \cap \bar{C} b>x$ then if $a$ and $b$ are not comparable, there may be no $c \in(A \cup B) \cap C$ that alone can dominate all elements in $(A \cup B) \cap \bar{C}$. So (OR) is not satisfied either.

So the weak optimistic dominance is a richer concept than the strong one.

\subsection{Refinement of partial preorders induced between subsets}

None of the relations presented in the above sections satisfies the property of Preadditivity, which considers that the common part of two sets should play no role in the comparison. A preadditive approach for comparing two sets $A$ and $B$ consists in eliminating the common part and then comparing $A \backslash B$ and $B \backslash A$. This is not a new idea (see [8] for a bibliography). In the following we consider the refinement of the weak optimistic dominance.

\section{Definition 4 ( Preadditive dominance)}

- strict preadditive dominance: $A \succ_{d}^{\text {wos }} B$ if and only if $A \neq B$ and $A \backslash B \succ_{\text {wos }}$ $B \backslash A$.

- loose preadditive dominance: $A \succeq_{d}^{w o l} B$ if and only if $A \backslash B \succeq_{w o l} B \backslash A$.

- strict order from $\succeq_{d}^{w o l}: A \succ_{d}^{w o l} B$ if and only if $A \succeq_{d}^{\text {wol }} B$ and $\neg\left(B \succeq_{d}^{\text {wol }} A\right)$.

These relations are respectively denoted by $\triangleright_{6}, \triangleright_{4}$ and $\triangleright_{5}$ in Halpern [8]. The two first relations are thoroughly studied in [23] and [24]. They coincide with $\succ_{\text {wos }}$ and $\succeq_{w o l}$ on disjoint sets. When $S$ is totally ordered, the relations $\succ_{d}^{w o l}$ and $\succ_{d}^{\text {wos }}$ coincide. Neither the relation $\succeq_{d}^{w o l}$, nor its strict part are transitive, as indicated by the following counterexample.

Example 6 Let $S=\left\{a_{1}, a_{2}, a_{3}, b_{1}, b_{2}, c\right\}$ with $a_{1} \sim a_{2} \sim b_{1}, a_{1}>c$ and $a_{3}>b_{2}$. Let $A=\left\{a_{1}, a_{2}, a_{3}\right\}, B=\left\{b_{1}, b_{2}\right\}, C=\left\{a_{1}, a_{2}, c\right\}$. We have $A \succeq_{d}^{\text {wol }} B$ but not $B \succeq_{d}^{\text {wol }} A, B \succeq_{d}^{\text {wol }} C$ but not $C \succeq_{d}^{\text {wol }} B$ but we don't have $A \succeq_{d}^{\text {wol }} C$.

The relation $\succ_{d}^{\text {wos }}$ seems to be more appropriate due to the following properties:

Proposition 4 The relation $\succ_{d}^{\text {wos }}$ is a strict partial order that satisfies:

- Strict compatibility with Inclusion (SCI): if $A \subset B$ then $B \succ_{d}^{\text {wos }} A$.

- Self-duality $(D)$ and Preadditivity $(P)$

- a weak form of Negligibility: If $A \cap B=A \cap C$ then (If $A \succ_{d}^{\text {wos }} B$ and $A \succ_{d}^{\text {wos }} C$ then $A \succ_{d}^{\text {wos }}(B \cup C)$ ).

- a weak form of Qualitativeness: If $A \cap B=A \cap C=B \cap C$ then (If $A \cup C \succ_{d}^{\text {wos }} B$ and $A \cup B \succ_{d}^{\text {wos }} C$ then $A \succ_{d}^{\text {wos }}(B \cup C)$ ).

Note that since $\succ_{d}^{w o s}$ is equal to $\succ_{\text {wos }}$ on disjoint sets, it satisfies (CCI), (CCC), (OR), CUT and CM as well.

The next property relates the optimistic dominance to the preadditive dominance.

Proposition $5 \succ_{d}^{\text {wos }}$ refines $\succ_{\text {wos }}$ and its dual variant: 
- If $A \succ_{\text {wos }} B$ then $A \succ_{d}^{\text {wos }} B$.

- If $\bar{B} \succ_{\text {wos }} \bar{A}$ then $A \succ_{d}^{\text {wos }} B$.

- If $A \succ_{d}^{\text {wos }} B$ then $A \succeq_{\text {wol }} B$ and $\bar{B} \succeq_{\text {wol }} \bar{A}$.

The preadditive dominance based on the weak optimistic dominance is thus welladapted to plausible reasoning with partially ordered knowledge bases. Note that the properties of Conditional Closure by Implication $(\mathrm{CCI})$ and Conditional Closure by Conjunction (CCC) are essential to extract a deductively closed set of most plausible formulae.

\subsection{From weak optimistic dominance to a partial order on elements}

Halpern [8] studied the problem to know if a preorder on $2^{S}$ can be generated by a preorder on $S$. The only known result deals with total preorders: If $\unrhd$ is a total preorder on $2^{S}$ that satisfies the properties of orderliness and qualitativeness, then there exists a total preorder $\geq$ on $S$ such that $\unrhd$ and $\succeq_{\text {wol }}$ coincide on $2^{S}$ (a similar result where one replaces these properties by stability for the union is already in [10], because in this case indeed a comparative possibility measure [9] is characterized by a complete preorder of possibility on $S$ ).

In the partial order case, if a strict order $\triangleright$ on $2^{S}$ is generated by a strict order $>$ on $S$, one must have $\{a\} \triangleright\{b\}$ whenever $a>b$. Conversely, suppose $\triangleright$ satisfies the properties of orderliness and negligibility and define the relation $a>_{\triangleright} b$ by $\{a\} \triangleright\{b\}$. Then, $A \succ_{\text {wos }} B$ means $\forall b \in B, \exists a \in A, a>_{\triangleright} b$ that is to say $\forall b \in B, \exists a \in$ $A,\{a\} \triangleright\{b\}$. We have:

- If $A \succ_{\text {wos }} B$ then $A \triangleright B$.

- Conversely, if $A \triangleright B$, it is easy to prove that $\forall b \in B, A \triangleright\{b\}$. But nothing proves that $\exists a \in A$ such that $\{a\} \triangleright\{b\}$.

So, the situation of partial orders is strikingly different from the case of total orders. Even equipped with the properties of orderliness and negligibility, a partial order on subsets is generally NOT characterized by its restriction on singletons.

Another way to induce a partial order on $2^{S}$ from a partial order $>$ on $S$ is to consider the partial order $>$ as a family of total orders $>^{i}$ extending (or compatible with) this partial order. Let $A$ and $B$ two subsets of $S$, and let $\triangleright^{i}$ denote the partial order on $2^{S}$ induced by $\succ^{i}$. Then two methods for building a partial order on $2^{S}$ can classically be proposed [25]:

Cautious principle considering all the total orders on $S$ compatible with $>: A \triangleright B$ iff $\forall i=1, \ldots, n, A \triangleright^{i} B$

Bold principle considering at least one total order on $S$ compatible with $>: A \triangleright B$ iff $\exists i, A \triangleright^{i} B$

It turns out that if we consider the family of total orders $>^{i}$ extending a partial order $>$ on $S$, the cautious principle enables the weak optimist dominance $\succ_{\text {wos }}$ to be recovered: 
Proposition 6 Let $A, B$ two subsets of $S$. We have:

$$
A \succ_{\text {wos }} B \Longleftrightarrow \forall i=1 . . n A \succ_{o}^{i} B
$$

As a consequence, a weak optimistic strict order on subsets is characterised by several total orderings on elements, not by a single partial order on elements. Given the properties satisfied by $\succ_{\text {wos }}$, this result clearly bridges the gap between the weak optimistic dominance and the partially ordered non-monotonic inference setting of Kraus, Lehmann and Magidor [20] interpreting the dominance $A \succ_{\text {wos }} B$ when $A \cap B=\varnothing$ as the default inference of $A$ from $A \cup B$.

Example 7 Let $(S,>)=\{a, b, c, d, e\}$ be the partially ordered set defined by $e>$ $c>a>d, c>b>d$. Let $>^{1},>^{2}$ be the two linear orders that extend the partial order $>$ defined by $e>^{1} c>^{1} b>^{1} a>^{1} d$ and $e>^{2} c>^{2} a>^{2} b>^{2} d$. Let $A=\{e, c\}, B=\{b, d\}$ and $C=\{a, d\}$ :

- $\forall i=1,2, \max (A)>^{i} \max (B)$, and it holds that $A \succ_{\text {wos }} B$.

- $\max (C)>^{2} \max (B), \max (B)>^{1} \max (C)$. Neither $C \succ_{\text {wos }} B$ nor $B \succ_{\text {wos }} C$.

\section{Representations of an epistemic state}

Let us first formalize the concept of epistemic state based on the notion of partial order, from a syntactic and semantic point of view. In the following, $V$ will denote a set of propositional variables, $\mathcal{L}$ a propositional language on $V$, and $\mathcal{K}$ a finite base of formulas built on $\mathcal{L}$.

\subsection{Syntactic representation}

From the syntactic point of view, we can view an epistemic state as a finite set of propositional formulas equipped with a partial preorder. Let $(\mathcal{K},>)$ be a partially ordered base of formulas. If $\phi$ and $\psi$ are two formulas of $\mathcal{K}, \psi>\phi$ is interpreted by " $\psi$ is more likely than $\phi$ " (typically the first one is more certain or plausible as the second one). It can more generally be interpreted in terms of "priority". If $\psi>\phi$ is viewed as a constraint, the presence of the likelihood relation can be a cause of inconsistency. For instance, it seems irrational to assert $\phi>\psi$ when $\phi \models \psi$. It can be regarded as a semantic contradiction.

In the particular case where the preorder is total, there is a alternative representation by means of a stratified base $\left(\mathcal{K}_{1}, \cdots, \mathcal{K}_{n}\right)$ where all the elements of $\mathcal{K}_{i}$ are set at the same priority level, and those of $\mathcal{K}_{i}$ are strictly preferred to those of $\mathcal{K}_{j}$ if $i>$ $j$. However, possibilistic logic [2] does not consider stratification as a strict ordering constraint. It interprets $\phi \in \mathcal{K}_{i}$ as assigning a minimal absolute level to $\phi$, that may fail to be its final one, i.e. $\phi$ can end up at some level $j>i$ in the totally ordered deductive closure (which represents an epistemic entrenchment relation). Therefore, the stratification of the base is never an additional source of inconsistency. On the contrary, if when $\psi \in \mathcal{K}_{i}$ and $\phi \in \mathcal{K}_{j}, j>i$ is understood as a constraint $\psi>\phi$, it means that the stratified knowledge base is viewed as a fragment of a likelihood relation (epistemic entrenchment or necessity measure). The complexity of finding the deductive closure is higher in the last situation due to the possibility of a semantic contradiction between the likelihood relation at the syntactic level and logical entailment. 


\subsection{Semantic representation}

Let $\Omega$ be the set of interpretations of $\mathcal{L}$. At the semantic level, suppose that an epistemic state is modelled by a partial preorder on the interpretations of a propositional language, $(\Omega, \triangleright)$. If $\omega$ and $\omega^{\prime}$ represent two elements of $\Omega$, the assertion $\omega^{\prime} \triangleright \omega$ is interpreted as $\omega^{\prime}$ being more plausible than $\omega$. In the knowledge representation literature, the main concern is often to extract the closed set of accepted beliefs $\mathcal{K}_{\triangleright}$ (or belief set) associated with $(\Omega, \triangleright)$. It is often defined as the deductively closed set of formulas whose models form the set $M(\Omega, \triangleright)$ of most plausible models. Our aim is to go further and to define a deductive closure which is a partial order induced by $(\Omega, \triangleright)$ on the language, $(\Omega, \triangleright)$ being itself induced by a partially ordered base $(\mathcal{K},>)$. The idea is to attach a semantics to $\phi>\psi$ in terms of a partial order on the interpretations, and then to build a partial order on $\mathcal{L}$ which is, as much as possible, in agreement with $(\mathcal{K},>)$. The question is thus to go from $(\mathcal{K},>)$ to $(\Omega, \triangleright)$ and back, namely:

From $(\mathcal{K},>)$ to $(\Omega, \triangleright)$ : Starting from a partially ordered base, the problem is to build a partial preorder on the set of interpretations of $\mathcal{K}$. A natural approach is to compare two interpretations $\omega$ and $\omega^{\prime}$ by comparing subsets of formulas of $\mathcal{K}$ built from these interpretations.

A first proposal is to compare two interpretations $\omega$ and $\omega^{\prime}$ by comparing the two subsets of formulas of $\mathcal{K}$ respectively satisfied by each of these interpretations. That is to say: $\omega^{\prime}$ is more plausible than $\omega$ if for each formula $\phi$ satisfied by $\omega$, there exists a formula preferred to $\phi$ and satisfied by $\omega^{\prime}$.

A dual proposal consists in comparing $\omega$ and $\omega^{\prime}$ by comparing the two subsets of formulas of $\mathcal{K}$ respectively falsified by each of these interpretations. That is to say: $\omega^{\prime}$ is more plausible than $\omega$ if for each formula $\phi^{\prime}$ falsified by $\omega^{\prime}$, there exists a formula falsified by $\omega$ preferred to $\phi^{\prime}$.

From $(\Omega, \triangleright)$ to $(\mathcal{L}, \succ)$ : Starting from a partial preorder on $\Omega$, the problem is to build a partial preorder on the set of the formulas of the language $\mathcal{L}$. To this end, it is natural to compare two formulas $\phi$ and $\phi^{\prime}$ by comparing subsets of interpretations built from these formulas. In the same way as above, a first proposal is to compare $\phi$ and $\psi$ by comparing the sets of models of these formulas. One can alternatively compare $\phi$ and $\psi$ by comparing their sets of counter-models, that is the models of $\neg \psi$ and $\neg \phi$.

In fact the choice between the two alternative approaches must be guided by the meaning of the relations on the families of sets. If $(\mathcal{K},>)$ is interpreted in terms of relative certainty as in possibilistic logic, it is natural to compare the subsets of falsified formulas of $\mathcal{K}$ for assessing the relative plausibility of interpretations. Indeed, an interpretation $\omega$ is all the less plausible as it violates more certain propositions.

In the same way, starting from a plausibility relation on the interpretations $(\Omega, \triangleright)$, we can express the idea of relative certainty $\phi \succ \psi$ on the language, by comparing sets of models of $\neg \psi$ and $\neg \phi$; for instance, in the case of a total order, a relation of comparative necessity, dual to comparative possibility, can be defined by $\phi \succ_{N} \psi$ iff $\neg \psi \succ_{o} \neg \phi^{1}$.

\footnotetext{
${ }^{1}$ relation $\succ_{0}$ is introduced in Definition 1.
} 
This approach was thoroughly studied within the possibilistic framework for completely ordered bases [2], but much less often in the partially ordered case [22].

Some questions will arise naturally from this research program:

- Is the partial preorder $\succ$ built on $\mathcal{L}$ from $(\Omega, \triangleright)$ compatible with $(\mathcal{K},>)$ ? A strict meaning of compatibility would require that $>$ is preserved and refined. Note that this is not the case in possibilistic logic if the lower bounds on certainty weights are not in conformity with classical deduction. Here again, it may happen that the relation $\succ$ on formulas induced from $\triangleright$ does not preserve the original ordering $(\mathcal{K},>)$, since the latter can be in conflict with semantic entailment, if supplied by some expert.

- Is the preorder built on $\Omega$ from $(\mathcal{K},>)$ unique? The answer is almost obviously no, as it depends on how the ordering $>$ is understood in terms of a relation between sets of models of formulas appearing in $K$.

- Is it still possible to use a principle of minimal commitment in order to select a complete preorder on $\Omega$ in a non arbitrary way? In possibilistic logic [2], this is the principle of minimal specificity that yields the least informative possibility distribution on $\Omega$ (akin to the most compact ranking in system $\mathrm{Z}$ [26]).

The two transformations: from $(\mathcal{K},>)$ to $(\Omega, \triangleright)$ and from $(\Omega, \triangleright)$ to $(\mathcal{L}, \succ)$ can be reduced to the problem of extending a partial order on a set $S$ to a partial order on the set of the subsets of $S$, discussed in Section 4.

\section{Optimistic dominance on partially ordered belief bases}

As in possibilistic logic, we assume that the relation $>$ expresses relative certainty, therefore we use the definitions based on falsified formulas. According to the previous sections, two approaches can be followed, using the weak optimistic dominance and its preadditive refinement. In the following, we consider both approaches consecutively. We do not consider the strong optimistic dominance as it allows to compare much less subsets, and we restrict here to strict dominance.

\subsection{Weak optimistic dominance semantics}

Let $(\mathcal{K},>)$ be a finite partially ordered set of formulas of the propositional language $\mathcal{L}$ build on $V . \mathcal{K}(\omega)$ (resp. $\overline{\mathcal{K}(\omega)}$ ) denotes the subset of formulas of $\mathcal{K}$ satisfied (resp. falsified) by the interpretation $\omega \in \Omega$. [ $\phi]$ denotes the set of the models of $\phi$, a subset of $\Omega$.

Definition $5[\operatorname{From}(\mathcal{K},>)$ to $(\Omega, \triangleright)] \forall \omega, \omega^{\prime} \in \Omega, \omega \triangleright_{\text {wos }} \omega^{\prime}$ iff $\overline{\mathcal{K}\left(\omega^{\prime}\right)} \succ_{\text {wos }} \overline{\mathcal{K}(\omega)}$

In the spirit of possibilistic logic, it defines the dominance on interpretations in terms of the violation of the most certain formulas. But here these formulas may be incomparable.

Definition $6\left[\operatorname{From}(\Omega, \triangleright)\right.$ to $\left.\left(\mathcal{L}, \succ_{N}\right)\right] \forall \phi, \psi \in \mathcal{L}, \phi \succ_{N} \psi$ iff $\overline{[\psi]} \triangleright_{\text {wos }} \overline{[\phi]}$. 
In the case of a total order, it would define a necessity relation on the language. The partially ordered deductive closure of $(\mathcal{K},>)$ is then defined by

$$
\mathcal{C}(\mathcal{K},>)_{\succ_{N}}=\left\{(\phi, \psi) \in \mathcal{L}^{2}: \phi \succ_{N} \psi\right\} .
$$

And we denote $(\phi, \psi) \in \mathcal{C}(\mathcal{K},>)_{\succ_{N}}$ by $\mathcal{K} \models{ }_{\text {wos }} \phi \succ_{N} \psi$. Besides, in agreement with [21], one may extract from $\mathcal{C}(\mathcal{K},>)_{\succ_{N}}$ the set of accepted beliefs when $\phi$ is known to be true as $\mathcal{A}_{\phi}(\mathcal{K},>)_{\succ_{N}}=\left\{\psi:(\phi \rightarrow \psi, \phi \rightarrow \neg \psi) \in \mathcal{C}(\mathcal{K},>)_{\succ_{N}}\right\}$. Note that these are generic definitions that make sense for any variant of the optimistic strict order on $\mathcal{K}$.

\section{Proposition 7}

- the relation $\triangleright_{\text {wos }}$ respects inclusion: If $\mathcal{K}(\omega) \subseteq \mathcal{K}\left(\omega^{\prime}\right)$ then $\omega \triangleright_{\text {wos }} \omega^{\prime}$ does not hold; and $\triangleright_{\text {wos }}$ is orderly too.

- If the relation $>$ is the strict part of a total preorder, possibilistic logic is recovered (order "best out" in [3]).

- If $\phi$ is a logical consequence of $\psi$, it does not hold that $\psi \succ_{N} \phi$.

$-\succ_{N}$ verifies the converse of the stability for intersection: if $\phi \wedge \chi \succ_{N} \psi \wedge \chi$, then $\phi \succ_{N} \psi$.

As a consequence, if the partial order on $\mathcal{K}$ violates the partial order induced by classical inference, $\succ_{N}$ will not refine it, but will correct it.

Example $8(\mathcal{K},>)=\{x>x \wedge y\}$. As usual, the four interpretations are denoted by $x y, x \bar{y}, \bar{x} y, \bar{x} \bar{y}$.

Clearly $\overline{\mathcal{K}(x y)}=\varnothing, \overline{\mathcal{K}(x \bar{y})}=\{x \wedge y\}, \overline{\mathcal{K}(\bar{x} y)}=\overline{\mathcal{K}(x y)}=\mathcal{K}$. Hence, $x y \triangleright_{\text {wos }}$ $\{x \bar{y}, \bar{x} y, \bar{x} \bar{y}\}$ and $x \bar{y} \triangleright_{\text {wos }}\{\bar{x} y, \bar{x} \bar{y}\}$. Then it is easy to see that $\mathcal{K} \models_{{ }_{\text {wos }}} x \succ_{N} y$ (since $[\neg y]=\{x \bar{y}, \bar{x} \bar{y}\} \triangleright_{\text {wos }}[\neg x]=\{\bar{x} y, \bar{x} \bar{y}\}$ but $\mathcal{K} \forall_{\text {wos }} y \succ_{N} x \wedge y$, since it does not hold that $[\neg x \vee \neg y]=\{\bar{x} y, x \bar{y}, \bar{x} \bar{y}\} \triangleright_{\text {wos }}[\neg y]=\{x \bar{y}, \bar{x} \bar{y}\}$.

If (by mistake) we set $\left(\mathcal{K}^{\prime},>\right)=\{x \wedge y>x\}$, note that we still have that $x y \triangleright_{\text {wos }}$ $\{x \bar{y}, \bar{x} y, \bar{x} \bar{y}\}$ but not $x \bar{y} \triangleright_{\text {wos }}\{\bar{x} y, \bar{x} \bar{y}\}$. Then

$\mathcal{K}^{\prime} \not \forall_{\text {wos }} x \wedge y \succ_{N} x$, that is, we correct this inconsistency via the semantics.

However, the fact, pointed out in Section 2, that a partial order over a power set cannot be characterized by a single partial order on the set of elements may cause some available pieces of knowledge in $(\mathcal{K},>)$ to be lost in $\mathcal{C}(\mathcal{K},>)_{\succ_{N}}$, as shown thereafter.

Example 9 Let $(\mathcal{K},>)=\{x, \neg x \vee y, x \wedge y, \neg x\}$ be a partially ordered base, where $>$ is the strict partial order given as follows: $\neg x \vee y>x \wedge y>\neg x$ and $x>\neg x$.

Let us apply the definitions 5 and 6:

- $\operatorname{From}(\mathcal{K},>)$ to $(\Omega, \triangleright):$ we obtain $x y \triangleright_{\text {wos }}\{\bar{x} y, x \bar{y}, \bar{x} \bar{y}\}$

- From $(\Omega, \triangleright)$ to $\left(\mathcal{L}, \succ_{N}\right)$ : we obtain $x \succ_{N} \neg x, x \wedge y \succ_{N} \neg x$ and $\neg x \vee y \succ_{N} \neg x$

but not $\neg x \vee y \succ_{N} x \wedge y$

We notice that, in the final order over formulas, $\neg x \vee y$ and $x \wedge y$ become incomparable. The reason is that some information has been lost when going from $(\mathcal{K},>)$ to $(\Omega, \triangleright)$. Indeed, if the strict partial order $>$ of the base $\mathcal{K}$ is interpreted as the strict part $\succ_{N}$ of a necessity ordering, applying Definition 6 enables the following constraints to be obtained: 
- Due to $\neg x \vee y \succ_{N} x \wedge y$ we must have $\bar{x} y \triangleright x \bar{y}$ or $\bar{x} \bar{y} \triangleright x \bar{y}$

- Due to $x \wedge y \succ_{N} \neg x$ we must have $x y \triangleright x \bar{y}$ and $x y \triangleright \bar{x} y$ or $x \bar{y} \triangleright \bar{x} y$ and $x y \triangleright \bar{x} \bar{y}$ or $x \bar{y} \triangleright \bar{x} \bar{y}$

- Due to $x \succ_{N} \neg x$ we must have $x y \triangleright \bar{x} y$ or $x \bar{y} \triangleright \bar{x} y$ and $x y \triangleright \bar{x} \bar{y}$ or $x \bar{y} \triangleright \bar{x} \bar{y}$

It is easy to see that these constraints imply that $x y \triangleright\{\bar{x} y, x \bar{y}, \bar{x} \bar{y}\}$ and $(\bar{x} y \triangleright x \bar{y}$ or $\bar{x} \bar{y} \triangleright x \bar{y})$. That is stronger than the partial order $\triangleright_{\text {wos }}$ and not representable by a single partial order.

One observes that the impossibility of representing the partial order $(\mathcal{K},>)$ by a partial ordering on interpretations is the cause for losing the piece of information $\neg x \vee$ $y \succ_{N} x \wedge y$. It suggests that the partially ordered deductive closure $\mathcal{C}\left(\mathcal{K},>_{\succ_{N}}\right.$ is too weak to account for semantic entailment in partially ordered knowledge bases.

\subsection{Preadditive semantics}

Under the preadditive semantics, the weak optimistic semantics in Definitions 5 and 6 is strengthened as follows:

Definition 7 Let $\omega, \omega^{\prime}$ be two interpretations:

- From $(\mathcal{K},>)$ to $(\Omega, \triangleright): \omega \triangleright_{d}^{\text {wos }} \omega^{\prime}$ iff $\mathcal{K}(\omega) \succ_{d}^{\text {wos }} \mathcal{K}\left(\omega^{\prime}\right)$.

- $\operatorname{From}(\Omega, \triangleright)$ to $\left(\mathcal{L}, \succ_{d}\right): \phi \succ_{d} \psi$ iff $[\phi] \triangleright_{d}^{\text {wos }}[\psi]$.

The notion of semantic consequence and deductive closure are defined similarly, replacing $\succ_{N}$ by $\succ_{d}$. The following results hold:

Proposition 8 It is clear that:

- the relation $\triangleright_{d}^{\text {wos }}$ strictly respects inclusion: If $\mathcal{K}(\omega) \subset \mathcal{K}\left(\omega^{\prime}\right)$ then $\omega^{\prime} \triangleright_{d}^{\text {wos }} \omega$.

- If $\phi$ is a proper logical consequence of $\psi$, then $\phi \succ_{d} \psi$.

- if $\chi \wedge(\phi \vee \psi)=\perp$ then $\phi \succ_{d} \psi$ implies $\phi \wedge \chi \succ_{d} \psi$.

- $\phi \wedge \chi \succ_{d} \psi \wedge \chi$ implies $\phi \succ_{d} \psi$

\section{Example 9 (continued)}

- $\operatorname{From}(\mathcal{K},>)$ to $(\Omega, \triangleright): x y \triangleright_{d}^{\text {wos }}\{\bar{x} y, x \bar{y}, \bar{x} \bar{y}\}$

- $\operatorname{From}(\Omega, \triangleright)$ to $\left(\mathcal{L}, \succ_{d}\right)$ :

- $x \succ_{d} x \wedge y \succ_{d} \neg x$

- $\neg x \vee y \succ_{d} x \wedge y \succ_{d} \neg x$

We notice that the relation $\succ_{d}$ has preserved and extended the initial strict partial order.

However, the relation $\succ_{d}$ does not always preserve the initial strict partial order as shown thereafter.

Example 10 Let $(\mathcal{K},>)=\{x, \neg x \vee \neg y, x \wedge y, \neg x\}$ be a partially ordered base, where $>$ is the strict partial order given as follows: $\neg x \vee \neg y>x \wedge y>\neg x$ and $x>\neg x$. 
- $\operatorname{From}(\mathcal{K},>)$ to $(\Omega, \triangleright)$ : we obtain $x \bar{y} \triangleright_{d}^{\text {wos }}\{\bar{x} y, x y, \bar{x} \bar{y}\}$

- From $(\Omega, \triangleright)$ to $\left(\mathcal{L}, \succ_{d}\right):$ we obtain $\neg x \vee \neg y \succ_{d} x \wedge y$, $\neg x \vee \neg y \succ_{d} \neg x, x \succ_{d} x \wedge y$

but not $x \wedge y \succ_{d} \neg x$.

Finally, let us consider the particular case of flat bases, interpreted as containing formulas that are all equivalent or all incomparable. The former case corresponds to classical logic. Suppose formulas in $\mathcal{K}$ are either incomparable or equivalent (for no $\phi, \psi \in \mathcal{K}$ do we have $\phi>\psi$ ). Then the set of logical consequences is no longer flat. The induced orderings are as follows.

- From (flat) $\mathcal{K}$ to $(\Omega, \triangleright): \omega^{\prime} \triangleright_{d}^{w o s} \omega$ iff $\mathcal{K}\left(\omega^{\prime}\right) \supset \mathcal{K}(w)$

- From $(\Omega, \triangleright)$ to $\left(\mathcal{L}, \succ_{d}\right): \phi \succ_{d} \psi$ iff $\forall \omega^{\prime} \in[\psi] \backslash[\phi], \exists w \in[\phi] \backslash[\psi]$ such that $\mathcal{K}(\omega) \supset \mathcal{K}\left(\omega^{\prime}\right)$.

Thus it is easy to see that, for flat bases, $\phi \succ_{d} \psi$ if and only if $\phi$ is a proper logical consequence of $\psi$, which enriches the semantics of classical logic.

\section{Towards syntactic inference with partially ordered belief bases}

Once the semantics of partially ordered belief bases and their deductive closure are well-defined, the next step is to devise a syntactic inference relation $\vdash$ that enables to directly build the ordered deductive closure $\mathcal{C}(\mathcal{K},>)_{\succ}$ from $(\mathcal{K},>$ ) (for a suitable choice of $\succ$ ) in agreement with the semantics, namely $(\mathcal{K},>) \vdash \phi \succ \psi$, whenever $(\phi, \psi) \in \mathcal{C}(\mathcal{K},>)_{\succ}$. It appears that this question has been little discussed in the partially ordered case, except by Halpern [8] and more recently by Benferhat and Prade [7], with very different approaches.

Several methods of inference from a partially ordered belief base have been proposed. Is is possible to:

1. map $(\mathcal{K},>)$ to a partially ordered set $\left(L_{\mathcal{K}},>\right)$ of absolute levels of certainty, and replace $(\mathcal{K},>)$ by a possibilistic knowledge base $B$ made of pairs $(\phi, \lambda), \lambda \in L_{\mathcal{K}}$, such that whenever $\phi>\psi,(\phi, \lambda),(\psi, \mu) \in B$ and $\lambda>\mu$. Then we can adapt the techniques of possibilistic logic to this setting.

2. consider a partial order as a family of total orders that extend it. So, a partially ordered base is seen as a set of (virtual) stratified bases.

3. reason directly with formulas $\phi>\psi$ in a suitable language.

4. reason in a classical way with consistent subsets of formulas extracted using the partial order.

The first approach was studied by Benferhat and Prade [7]. Let $\left(L_{\mathcal{K}},>\right)$ be a finite ordered set associated with $\mathcal{K}$ by a homomorphism $\iota: \mathcal{K} \rightarrow L_{\mathcal{K}}$ such that $\phi \geq \psi \in \mathcal{K}$ iff $\iota(\phi) \geq \iota(\psi)$. Let us denote $\{\mu: \mu \geq \lambda\}$ by $\lambda^{\uparrow}$. The inequality $\lambda_{1} \geq \lambda_{2}$ in $\left(L_{\mathcal{K}}, \geq\right)$ is encoded by $A_{2} \vee \neg A_{1}$ with $A_{i}=\lambda_{i}^{\uparrow}$, and the pair $(\phi, \lambda)$ is encoded by $\neg A \vee \phi$, with $A=\lambda^{\uparrow}$. Then classical propositional deduction can be used.

Actually, there is a more direct way to apply possibilistic logic to the partially ordered case. It is well-known that in standard possibilistic logic $B \vdash(\phi, \lambda) \Longleftrightarrow B_{\lambda} \vdash$ $\phi$ where $B_{\lambda}$ is the set of formulas with weights at least $\lambda$. In the partially ordered case, 
we could define, when $\psi \in \mathcal{K}, \mathcal{K} \vdash \phi \succ \psi$ by $\mathcal{K}_{\psi}^{>} \vdash \phi$ where $\mathcal{K}_{\psi}^{>}=\{\alpha \in \mathcal{K}: \alpha>\psi\}$ and likewise $\mathcal{K} \vdash \phi \succeq \psi$ by $\mathcal{K}_{\bar{\psi}}^{\geq} \vdash \phi$ where $\mathcal{K}_{\bar{\psi}}^{\geq}=\{\alpha \in \mathcal{K}: \psi \ngtr \alpha\}$. For instance if statements $\phi_{i}>\psi_{i}$ in $(K,>)$ are interpreted on the set $2^{\Omega}$ by $\overline{\left[\psi_{i}\right]} \triangleright \overline{\left[\phi_{i}\right]}$ where the relation $\triangleright$ satisfies Negligibility and Orderliness, it does hold that $[\psi] \triangleright \overline{[\phi]}$ whenever $\mathcal{K}_{\psi}^{>} \vdash \phi$. A particular case occurs when $\triangleright$ is $\succ_{\text {wos }}$.

However, as shown in Example 9, if the consequence $\phi \succ \psi$ is interpreted as $\phi \succ_{N}$ $\psi$ using Definitions 5 and 6 (that is,via a partial order on interpretations derived from $(K,>)$ ) it may fail to hold that $\phi \succ_{N} \psi$ whenever $\mathcal{K}_{\psi}^{>} \vdash \phi$ (in Example 9, $\phi>$ $\psi$ appears in $(K,>)$, and is absent from the semantic closure). This fact indicates a weakness in the semantics based on a partial order on interpretations, as opposed to a more complex semantics based on the partial ordering on subsets of interpretations reflecting $(K,>)$.

The second approach is described by Yahi and al [25]. $(\mathcal{K},>)$ is viewed as a set of possible stratifications of $\mathcal{K}$. So $\psi \geq \phi$ of $(\mathcal{K},>)$ means that $\psi$ is more certain than $\phi$ (in the sense of possibilistic logic) in all the stratified bases compatible with $(\mathcal{K},>)$. Results in the previous section indicate the strong link between this view and the weak optimistic relation $\succ_{\text {wos }}$ (hence its dual $\succ_{N}$ and their preadditive refinement $\succ_{d}$ ). Note that in the first approach [7], the partial order on $L_{\mathcal{K}}$ is actually viewed as a set of possible total orders. The weights are symbolic in the sense of being partially unknown quantities on a totally ordered scale. To use this approach in practice may turn out to be difficult, because the set of total extensions of a given partial order may be large.

In the third approach, the problem is to consider expressions of the form $\phi \succ \psi$ as the basic syntactic entities of the language encoding the preferences. It requires a higher order language for handling atomic propositions of the form $\phi \succ \psi$, their conjunctions, disjunctions and negations, with specific axioms for describing properties of the relation $>$. For instance, with the axiom: if $\psi \models \phi$ then $\neg(\psi \succ \phi)$, semantical contradictions will be found, thus enabling to repair the partially ordered base. This approach, which is the most natural one, goes back to Lewis [9] conditional logics (see also Hájek [27], p. 212) in the case of total preorders, for possibility theory). Halpern [8] has outlined such a logic to handle the relation $\succ_{\text {wos }}$. This is certainly the most general approach with the richest language. Especially it would readily allow for a semantics in terms of a partial order over the set of subsets of interpretations of the language, which would obviate difficulties pointed out by Examples 9 and 10 when we use a partial ordering on interpretations. However only a subset of the consequences $(K,>)$ from a set of $\phi \succ \psi$ statements will correspond to the (properly defined) semantic closure $\mathcal{C}(\mathcal{K},>)$ (since, for instance, the latter does not contain disjunctions of such statements).

In the fourth approach [22], the partial order on $\mathcal{K}$ is just used to select preferred consistent subsets of formulas, and the deductive closure is a classical set of accepted beliefs. So, as pointed out in Benferhat and Yahi [28], the deductive closure of a partially ordered base $(\mathcal{K},>)$ is just a deductively closed set (in the classical sense), obtained from preferred subbases. Then the inference $(\mathcal{K},>) \vdash \phi$ is defined by: $\phi$ is consequence of all the preferred subsets of formulas. The notion of preference can be defined in various ways based on the partial order. This order between formulas is to some extent lost by the process of inference. In particular this kind of approach reduces to classical inference when $\mathcal{K}$ is classically consistent. By construction, this approach 
does not enable to deduce preferences between formulas, but essentially extracts accepted beliefs.

In the future, we plan to investigate whether or not the above syntactic inference schemes are sound (and if possible complete) with respect to our notion of partialorder-driven semantic closure. We have already noticed that a semantics based on a single partial ordering over interpretations may be problematic as seen in Example 9. This result motivates the use of a modal-like language with formulas of the form $\phi>\psi$, $\phi \geq \psi$, or $\phi \sim \psi$ with relational semantics on the powerset of the set of interpretations of the language where $\phi, \psi$ are expressed, whereby $\phi>\psi$ is viewed as a relation between $\overline{\left[\psi_{i}\right]}$ and $\overline{\left[\phi_{i}\right]}$, etc. Then the properties of the semantic relation can be used as inference rules at the syntactic level. However, one may wish to restrict the inference machinery to consequences of the form $\phi \succ \psi$ and $\phi \succeq \psi$.

\section{Conclusion}

The issue addressed in this paper concerns the extension of possibilistic logic when formulas weighted by certainty levels are replaced by a partial order on the belief base. Defining proper semantics for such partially ordered bases requires the study of how to go from a partial order on elements to a partial order on subsets of such elements and conversely. Some preliminary results are offered in this paper. They indicate that many important concepts in the case of complete orders have several non equivalent definitions in the partial case. When going from a partial order on a set to a partial order on its subsets, it seems that the weak optimistic relation possesses the best properties. Moreover it seems that a straightforward adaptation of possibilistic logic to the partial order setting is not possible.

The question then becomes the one of finding the most natural understanding of a base partially ordered in terms of relative certainty. Our paper explains how to go from formulas to models and back, thus defining a semantic notion of deductive closure. We indicate that the expressive power of a partial order on the set of interpretations is limited, and one must stick to a partial order on its power set, or alternatively a set of total orders on the set of interpretations, to be on the safe side. Existing works proposing proof methods have been reviewed, but they all consider different points of view on the definition of inference in the partially ordered context, sometimes with unclear semantics. In contrast, our purpose is to eventually define a semantic closure that preserves and extends the partial order on $\mathcal{K}$ to the whole language, while correcting the initial assessment to make it comply with the classical deduction. Once this issue has been clarified, we have to choose an appropriate syntax, an axiomatization and a syntactic inference method. Some hints are provided above, but this is left for further research.

This work has potential applications for the revision and the fusion of beliefs, as well as preference modeling [29].

\section{References}

1. Rescher, N.: Plausible Reasoning. Van Gorcum, Amsterdam (1976) 
2. Dubois, D., Lang, J., Prade, H.: Possibilistic logic. In Gabbay, D., Hogger, C., Robinson, J., Nute, D., eds.: Handbook of Logic in Artificial Intelligence and Logic Programming, Vol. 3. Oxford University Press (1994) 439-513

3. Benferhat, S., Cayrol, C., Dubois, D., Lang, J., Prade, H.: Inconsistency management and prioritized syntax-based entailment. In Bajcsy, R., ed.: Proc. of the $13^{\text {th }}$ IJCAI, Chambéry, France, Morgan-Kaufmann (1993) 640-645

4. Dubois, D., Prade, H.: Epistemic entrenchment and possibilistic logic. Artificial Intelligence 50 (1991) 223-239

5. Benferhat, S., Dubois, D., Kaci, S., Prade, H.: Logique possibiliste et fusion d'informations. Technique et Science Informatique 22 (2003) 1035-1064

6. Dubois, D., Prade, H., Schockaert, S.: Régles et métarègles en théorie des possibilités. Revue d'Intelligence Artificielle 26 (2012) 773-793

7. Benferhat, S., Prade, H.: Encoding formulas with partially constrained weights in a possibilistic-like many-sorted propositional logic. In Kaelbling, L.P., Saffiotti, A., eds.: IJCAI, Professional Book Center (2005) 1281-1286

8. Halpern, J.Y.: Defining relative likelihood in partially-ordered preferential structures. Journal of Artificial intelligence Research 7 (1997) 1-24

9. Lewis, D.: Counterfactuals and comparative possibility. Journal of Philosophical Logic 2 (1973) 418-446

10. Dubois, D.: Belief structures, possibility theory and decomposable confidence measures on finite sets. Computers and Artificial Intelligence (Bratislava) 5 (1986) 403-416

11. Alchourrón, C.E., Gärdenfors, P., Makinson, D.: On the logic of theory change: partial meet contraction and revision functions. Journal of Symbolic Logic 50 (1985) 510-530

12. Fishburn, P.: Interval Orderings. Wiley, New-York (1987)

13. Benferhat, S., Dubois, D., Prade, H.: Towards a possibilistic logic handling of preferences. Appl. Intell. 14 (2001) 303-317

14. Kaci, S., van der Torre, L.: Reasoning with various kinds of preferences: Logic, nonmonotonicity and algorithms. Annals of Operations Research 163 (2008) 89-114

15. Kaci, S.: Working With Preferences: Less Is More. Springer (2012)

16. Fishburn, P.C.: The axioms of subjective probability. Statistical Science 1 (1986) 335-358

17. Dubois, D., Fargier, H., Prade, H.: Possibilistic likelihood relations. In: Proceedings of 7th International Conference on Information Processing and Management of Uncertainty in Knowledge-based Systems (IPMU'98), Paris, Editions EDK (1998) 1196-1202

18. Friedman, N., Halpern, J.Y.: Plausibility measures: A user's guide. In: Proc of the Eleventh Annual Conference on Uncertainty in Artificial Intelligence, Montreal, Quebec, August 1820. (1995) 175-184

19. Dubois, D., Prade, H.: Numerical representations of acceptance. In: Proc of the Eleventh Annual Conference on Uncertainty in Artificial Intelligence, Montreal, Quebec, August 1820. (1995) 149-156

20. Kraus, S., Lehmann, D., Magidor, M.: Nonmonotonic reasoning, preferential models and cumulative logics. Artificial Intelligence 44 (1990) 167-207

21. Dubois, D., Fargier, H., Prade, H.: Ordinal and probabilistic representations of acceptance. J. Artif. Intell. Res. (JAIR) 22 (2004) 23-56

22. Benferhat, S., Lagrue, S., Papini, O.: Reasoning with partially ordered information in a possibilistic logic framework. Fuzzy Sets and Systems 144 (2004) 25-41

23. Cayrol, C., Royer, V., Saurel, C.: Management of preferences in assumption-based reasoning. In Yager, R., Bouchon, B., eds.: Advanced methods in AI. Lecture notes in computer science 682. Springer Verlag (1992) 13-22

24. Geffner, H.: Default reasoning: Causal and Conditional Theories. MIT Press (1992) 
25. Yahi, S., Benferhat, S., Lagrue, S., Sérayet, M., Papini, O.: A lexicographic inference for partially preordered belief bases. In Brewka, G., Lang, J., eds.: KR, AAAI Press (2008) 507-517

26. Pearl, J.: System Z: A natural ordering of defaults with tractable applications to default reasoning. In Vardi, M., ed.: Proc. of the $3^{\text {rd }}$ Conference of Theoretical Aspects of Reasoning about Knowledge, Morgan-Kaufmann (1990) 121-135

27. Hájek, P.: The Metamathematics of Fuzzy Logics. Kluwer Academic (1998)

28. Benferhat, S., Yahi, S.: Etude comparative des relations d'inférence à partir de bases de croyance partiellement ordonnées. Revue d'Intelligence Artificielle 26 (2012) 39-61

29. Dubois, D., Prade, H., Touazi, F.: Conditional preference nets and possibilistic logic. In van der Gaag, L.C., ed.: Symbolic and Quantitative Approaches to Reasoning with Uncertainty. Volume 7958 of Lecture Notes in Computer Science., Springer (2013) 181-193 